Relations industrielles

Industrial Relations

\title{
The International Trade Union Movement, by Prof. J.P. Windmuller, Deventer, Boston, London, Frankfurt, Antwerp, Kluwer, 1980, 174 pp.
}

\section{Gérard Dion}

Volume 36, numéro 2, 1981

URI : https://id.erudit.org/iderudit/029170ar

DOI : https://doi.org/10.7202/029170ar

Aller au sommaire du numéro

Éditeur(s)

Département des relations industrielles de l'Université Laval

ISSN

0034-379X (imprimé)

1703-8138 (numérique)

Découvrir la revue

Citer ce compte rendu

Dion, G. (1981). Compte rendu de [The International Trade Union Movement, by Prof. J.P. Windmuller, Deventer, Boston, London, Frankfurt, Antwerp, Kluwer, 1980, 174 pp.] Relations industrielles / Industrial Relations, 36(2), 455-456.

https://doi.org/10.7202/029170ar

Tous droits réservés @ C Département des relations industrielles de l'Universite Laval, 1981
Ce document est protégé par la loi sur le droit d'auteur. L'utilisation des services d'Érudit (y compris la reproduction) est assujettie à sa politique d'utilisation que vous pouvez consulter en ligne.

https://apropos.erudit.org/fr/usagers/politique-dutilisation/ 
pension ou cause de rupture, les congés payés, la mise à pied économique. Ce chapitre trouve dans l'ouvrage de Jean-Marc Béraud - enseignant à l'Institut de Droit du Travail et de la Sécurité sociale de Lyon, dont Jean Pélissier est le (jeune) directeur honoraire - publié chez le même éditeur et dans la même «Bibliothèque de Droit du Travail et de la Sécurité sociale» dirigée par Gérard LyonCaen, le texte actuel de référence qui faisait défaut.

Traitement complet et nouveau d'un thème majeur du Droit du travail, le livre de J.-M. Béraud sur «La suspension du contrat de travail», et qui porte en sous-titre "Essai d'une théorie générale», est formé de deux parties: "La suspension du contrat de travail» et «Les effets de la suspension du contrat de travail», chacune divisée en plusieurs titres, chapitres et sections.

Ouvrage de qualité remarquable, ainsi que Jean Pélissier l'avait noté avec justesse dans sa préface, le travail de J.-M. Béraud révèle une personnalité affirmée, et dont ce premier texte est plus qu'une promesse: une consécration.

Je voudrais signaler, in fine, à propos et aux côtés des arrêts français commentés par G. Lyon-Caen et J. Pélissier, la parution du volume 2 de l'International Labour Law Reports, initiative dont j'avais loué l'excellence dans la note parue dans le $\mathrm{n}^{\circ} 4-1979$ de cette même Revue (pp. 825-826). Ce nouveau tome couvre des jugements s'échelonnant du $1^{\text {er }}$ octobre 1975 au 30 septembre 1976, et intervenus en Autriche (Walter Schwarz), en Belgique (Roger Blanpain), France (J.-M. Verdier et J.-C. Javillier), Allemagne fédérale (Thilo Ramm), Grande-Bretagne (Lord Wedderburn, M. Freedland, P.L. Davies), Israël (Zvi H. Bar-Niv et Ruth Ben Israel), Italie (Tiziano Treu), Japon (Yasuhiko Matsuda), Pays-Bas (J.A.O.M. Van Aerde), Suède (Olof Berquist) et États-Unis (Benjamin Aaron).

Ai-je besoin encore de dire que cet ouvrage de prestige est un instrument de travail indispensable pour tout pratiquant et ensei- gnant de Droit du travail, et faire part de mon souhait profond que le Canada n'en soit, désormais, plus absent?

Dimitri WEISS

Université du Maine et

Université de Paris I Panthéon - Sorbonne

The International Trade Union Movement, by Prof. J.P. Windmuller, Deventer, Boston, London, Frankfurt, Antwerp, Kluwer, 1980, 174 pp.

Depuis plus de trente ans, l'auteur s'intéresse au mouvement syndical international. Déjà, en 1954, il avait publié American Labor and the International Labor Movement 1940-1953 qui était le résultat de plusieurs années de recherches pour la préparation de sa thèse de doctorat. Il a continué à suivre de près l'évolution des groupements, à garder des contacts avec leurs principaux responsables et à tenir à jour sa documentation. Il est certainement l'une des rares personnes les mieux informées sur un pareil sujet.

Dans cet ouvrage, assez succinct, qui est la reprise de la monographie déjà parue dans l'International Encyclopedia for Labour Law and Industrial Relations (Kluwer), l'auteur, sans ennuyer le lecteur avec un apparat scientifique inutile, présente tout ce que l'on doit connaître sur le mouvement syndical international si l'on veut pouvoir se démêler dans un tel labyrinthe.

Les trois premiers chapitres abordent l'arrière-plan historique: avant 1919 (ch. I); 1919-1939 (ch. II) et depuis 1939 (ch. III). Les trois chapitres suivants sont consacrés aux grandes organisations syndicales internationales: la Confédération internationale des syndicats libres (ch. IV); la Fédération syndicale mondiale (ch. V) et la Confédération mondiale du travail (ch. VI). Dans les deux autres chapitres, l'auteur traite des Fédérations régionales indépendantes ou groupements assimilés (ch. VII) et des Secrétariats professionnels internationaux (ch. VIII). Le tout est suivi d'une brève, mais substantielle 
bibliographie. On y trouve, en outre, seize tableaux fournissant des détails (liste des affiliés, membres, distribution géographique, etc.) sur les diverses organisations ainsi qu'une nomenclature des sigles par lesquels elles sont communément désignées.

La présentation des chapitres touchant les organisations suit un plan uniforme: a) l'organisation et la structure (effectifs, organisme de direction, secrétariat, finances, organisations régionales); b) les objectifs, la politique et les activités; c) les relations avec les autres groupements.

Un des aspects intéressants de l'ouvrage, c'est que l'auteur ne se contente pas d'exposer ce que l'on pourrait retrouver dans la littérature officielle des groupements et qui est souvent marqué par des visées de propagande, mais il sait faire la part des choses et explique ce qui en est dans la réalité.

\section{Gérard DION}

Université L.aval

Les femmes et le syndicalisme, par Julie White, Conseil consultatif canadien de la situation de la femme, Ottawa, Centre d'édition du gouvernement du Canada, 1980, 147 pp.

Dans son étude Les femmes et le syndicalisme, effectuée pour le compte du Conseil consultatif canadien de la situation de la femme, Julie White se propose d'une part d'évaluer la situation des femmes à l'intérieur du mouvement syndical canadien et, d'autre part, d'analyser l'impact du syndicalisme sur les conditions de travail des femmes.

Une telle synthèse était nécessaire: l'action grandissante des femmes au sein des syndicats ainsi que les préoccupations du mouvement syndical dans son ensemble face aux revendications féminines ont amené cette question sur la place publique et ont fait ressentir encore plus cruellement l'absence de synthèse et d'analyse des données sur cette question.
Au cours des dernières années, la syndicalisation des femmes s'est accrue plus rapidement que leur participation au monde du travail. Ainsi, «de 1966 à 1976 le pourcentage de travailleuses syndiquées est passé de 16,3 à $26,8 \%$ » (p. 26). On note aussi que la syndicalisation des femmes a augmenté quatre fois plus rapidement que celle des hommes. Il s'agissait bien sûr de combler un retard historique et la syndicalisation du secteur public y compris celle des secteurs traditionnellement féminins que sont l'éducation et la santé expliquent cette forte croissance du syndicalisme parmi les femmes. Malgré cette hausse prodigieuse, il n'en demeure pas moins que les femmes sont toujours proportionnellement moins syndiquées que les hommes; en effet, à l'échelle canadienne, $27 \%$ des travailleuses sont syndiquées, alors que $43 \%$ des travailleurs le sont.

Un tel écart amène l'auteur à s'interroger sur les motifs du plus faible taux de syndicalisation des femmes. Rejetant d'emblée les explications d'ordre idéologique (attitudes des femmes ou des syndicats) Julie White rappelle que les secteurs où traditionnellement la syndicalisation a été la plus développée, notamment chez les cols bleus, sont aussi des secteurs à faible participation féminine. Inversement, elle note une forte participation des femmes à la main-d'oeuvre de secteurs tels le commerce, les finances et les services, secteurs peu ouverts à la syndicalisation. La taille de l'entreprise a aussi un impact sur le taux de syndicalisation. Aux fins de l'étude, une compilation spéciale de Travail-Canada portant sur les établissements de la fabrication en 1977 (p. 56) illustre que plus un établissement est important, plus élevé risque d'être la syndicalisation. Or, les ouvrières de la fabrication ont tendance à se concentrer dans des entreprises de petites dimensions d'où une syndicalisation moins développée.

L'auteur aborde aussi la question controversée des avantages de la syndicalisation répondant par là à certaines assertions parfois véhiculées dans le mouvement féministe ayant trait à l'inutilité du syndicalisme pour les femmes. Critiquant plus particulièrement 